\title{
Nucleus Polposus Embolism Causing Anterior Spinal Artery Occlusion: A Rare but Possible Cause of Fibrocartilaginous Embolic Myelopathy
}

\author{
MAYANK GUPTA, MS, HARVINDER SINGH CHHABRA, MS \\ Department of Spine Surgery, Indian Spinal Injuries Centre, Sector C, Vasant Kunj, Delhi, India, PIN 110070
}

\begin{abstract}
Background: Fibrocartilaginous embolic myelopathy (FCEM) is a rare cause of spinal cord infarction. It has been confirmed by autopsy in postmortem cases. Herein we describe a case of FCEM diagnosed based on clinical grounds.

Methods: A 58-year-old man presented with acute onset of complete paraplegia with bladder and bowel involvement developing a few hours after a trivial trauma. There were no upper motor neuron signs. His magnetic resonance imaging (MRI) was suggestive of longitudinally extensive transverse myelitis from T5 to the conus. There was left paracentral disc protrusion at the T4-T5 level. However, no features of inflammatory, infectious, or autoimmune etiology were found on history, on examination, or in blood or cerebrospinal fluid analysis, and there was no contrast enhancement on MRI.

Results: A diagnosis of anterior spinal artery occlusion was made based on clinical examination with sparing of posterior column sensations in the lower limbs, predominant involvement of anterior half of the spinal cord on MRI, and accompanying new onset of back pain with rapid symptom progression to nadir as opposed to inflammatory etiology. Fibrocartilaginous embolism was suspected after ruling out all other causes of vascular compromise and presence of disc herniation at T4-T5. He was managed with rehabilitation and showed no signs of recovery.

Conclusion: FCEM, though rare, should be kept in mind as a differential diagnosis of acute medical myelopathy when no other cause can be identified.

Complications

Keywords: fibrocartilaginous embolic myelopathy, nucleus polposus embolism, anterior spinal artery syndrome
\end{abstract}

\section{INTRODUCTION}

Fibrocartilaginous embolic myelopathy (FCEM) was first described by Naiman ${ }^{1}$ in 1961. It involves migration of the nucleus polposus material into the vessels supplying the spinal cord, resulting in embolic infarction. It can be confirmed only by histopathology at autopsy and has been reported in 41 such cases. ${ }^{2}$ It is difficult and rare to suspect this diagnosis clinically in vivo, and hence it is usually underrecognized. FCEM has also been described in animals, most commonly in dogs, where it is one of the most common causes of ischemic myelopathy. ${ }^{3,4}$

We report a rare case of FCEM diagnosed prospectively based on clinical findings.

\section{CASE PRESENTATION}

A 58-year-old male presented with sudden onset of weakness in the bilateral lower limbs, numbness in the lower half of body with bladder and bowel involvement in the form of urinary retention, hesitancy, and constipation 6-8 hours following a trivial trauma due to tripping from a flight of 2-3 stairs. There was mild upper back pain that subsided a few hours after the trauma with analgesics. He was able to walk normally after the traumatic incident. The weakness developed spontaneously at night, first in the right lower limb followed by the left lower limb, within a few hours. The numbness followed the same pattern. This was followed by urinary retention. There were no loss of consciousness, impaired mental activity, visual disturbances, past history of seizure disorder, or previous episodes of back pain or radiculopathy. There was no history of fever or any recent infection. There was no history of smoking, diabetes mellitus, or any other comorbidity. His general physical examination was within normal limits. Spine examination revealed no tenderness or any abnormal finding. His higher mental functions, cranial nerve examination, and 
upper limb neurology were normal. The paralysis in the lower limbs was complete, lower motor neuron type. The sensations of pain, temperature, and crude touch were absent below the T7 dermatomal level with preservation of posterior column sensations of vibration, proprioception, and position sense. All reflexes below the level were absent. Perianal sensations and voluntary anal contraction were completely absent. He was catheterized, and further investigations were performed to identify the cause of paraplegia.

Magnetic resonance imaging (MRI) of the spine revealed diffuse intramedullary hyperintensity extending from T5 to the conus region with mild cord edema, involving mainly the anterior half of the cord on T2-weighted imaging and short T1 inversion recovery, and was isointense on $\mathrm{T} 1$, suggestive of longitudinally extensive transverse myelitis or demyelinating disease (Figures $1 \mathrm{a}-\mathrm{d}$ and $2 \mathrm{a}-\mathrm{c}$ ). On axial sections, there was a left-sided paracentral disc protrusion at the T4-T5 level (Figure 2a). Degenerative disc changes were also found in the cervical region and the T11-T12 level (Figure 1). Postcontrast MRI revealed no abnormal cord enhancement and no evidence of leptomeningeal enhancement, ruling out inflammatory or infectious etiology (Figure 3). Diffusion-weighted imaging (DWI) of the spine was done, suspecting vascular etiology. It revealed a diffuse hyperintense signal in the cord extending from T5 to the conus with diffusion restriction and appeared hypointense on apparent diffusion coefficient (ADC), suggestive of an infarct in the anterior spinal artery (ASA) distribution. Further investigations were done to rule out autoimmune, inflammatory, or infectious etiologies. MRI of the brain was normal; visual evoked potentials and optical coherence tomography of the retinal nerve fiber layer were normal, ruling out neuromyelitis optica (NMO); and a cerebrospinal fluid (CSF) study was done and was also normal, ruling out inflammatory or infectious causes. CSF protein level was $59.1 \mathrm{mg} / \mathrm{dl}$. There was no pleocytosis or raised immunoglobulin $\mathrm{G}(\mathrm{IgG})$ index in the CSF. On blood investigations, markers of autoimmune or inflammatory etiologies were negative. Anti-NMO, anti-myelin oligodendrocyte glycoprotein antibodies, serum acetyl cholinesterase levels, antinuclear antibody, and cytoplasmic or perinuclear anti-neutrophilic cytoplasmic antibodies were negative. Procoagulant work-up to discover thromboembolic etiology, such as antiphospholipid antibody, protein $\mathrm{C}, \mathrm{S}$, lupus anticoagulant, anticardiolipin antibody, and beta-2 glycoprotein, were negative. The patient's electrocardiogram and echocardiography were normal. After exhaustive investigations and literature search, a diagnosis of nucleus polposus embolism from the T4-T5 disc causing ASA syndrome was made according to the criteria for clinical diagnosis proposed by AbdelRazek et al. ${ }^{2}$ These criteria suggest to first make a clinical diagnosis of myelopathy and then to exclude compressive or traumatic etiology by history and imaging. The third step is to exclude inflammatory etiology by CSF findings and contrast-enhanced MRI. The fourth step is to establish vascular cause based on examination and MRI. Other minor criteria proposed were new onset of back pain and symptom progression to nadir within 4-8 hours. There should also be no aortic pathology or other vascular risk factors or the presence of disc protrusion at or near the infarction. Our approach was based on these steps, and FCEM was concluded to be the most possible etiology. DWI and digital subtraction angiography are not included in these criteria and hence are not essential to diagnose FCEM. Based on the above steps, an algorithm has been devised, shown in Figure 4.

The patient was started on intravenous methylprednisolone for 5 days for presumed transverse myelitis while the diagnostic work-up was being done, and reports were awaited but showed no improvement. He was ultimately managed conservatively with rehabilitation and a physiotherapy program. Bowel training was initiated, and clean intermittent catheterization was advised for bladder management. The patient continues to be in followup and has shown no signs of neurological recovery even after 2 years. The patients and/or their families were informed that data from the case would be submitted for publication and gave their consent.

\section{DISCUSSION}

Vascular causes account for $5 \%-8 \%$ of the cases of acute myelopathies. ${ }^{5}$ Common causes of spinal cord infarction include thrombotic atherosclerotic disease, aortic dissection, surgical complication, vascular malformations in the spinal cord, vasculitis (eg, polyarteritis nodosa), and cardiac embolism. ${ }^{6}$ Fibrocartilaginous embolism (FCE) causing spinal cord infarction is a rare differential. The exact incidence is not known, as it is commonly labeled as idiopathic transverse myelitis, depending mainly on 


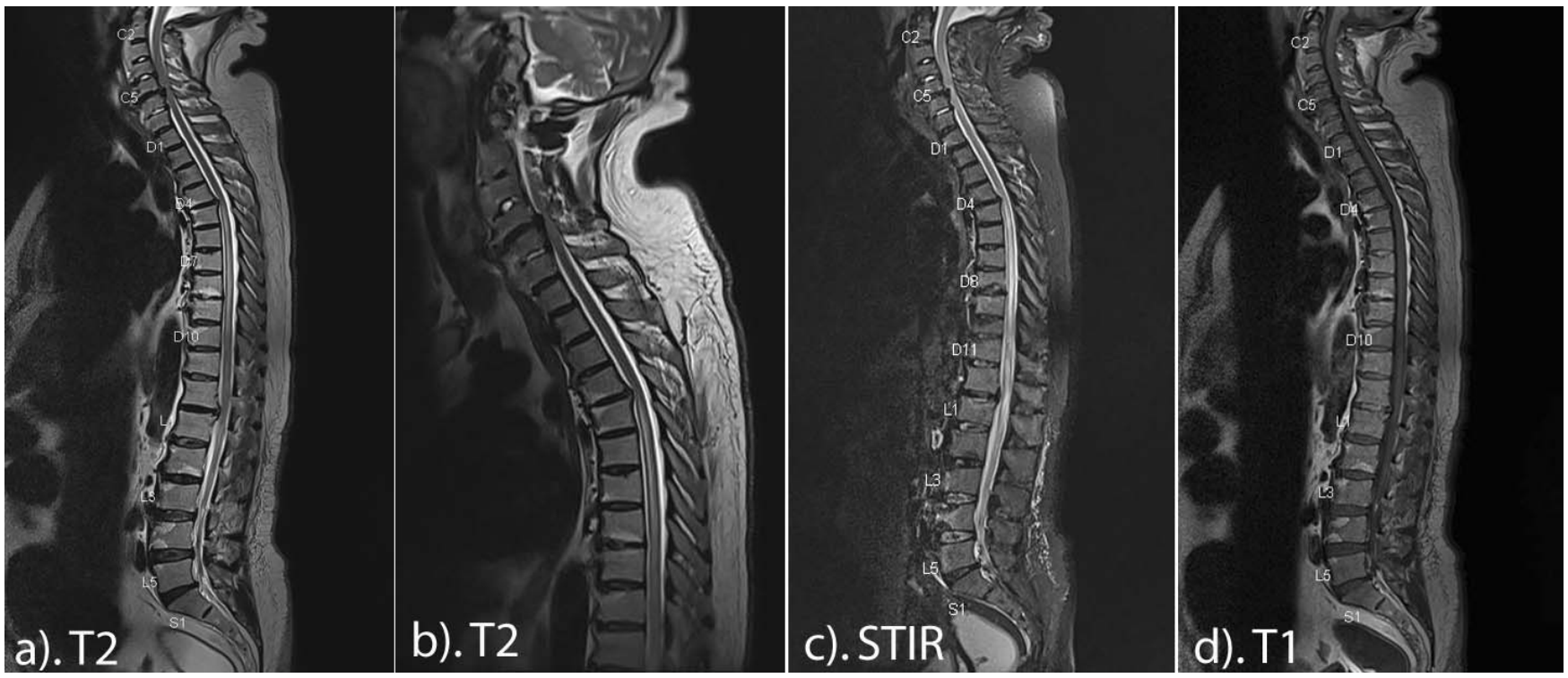

Figure 1. Sagittal images of the spine showing intramedullary hyperintensity extending from T5 to conus region with mild cord edema on T2 and short T1 inversion recovery images $(\mathrm{a}-\mathrm{c})$ that is isointense on $\mathrm{T} 1(\mathrm{~d})$.

the MRI image when no cause has been identified. ${ }^{7}$ It is found at an average of 22 years in young patients and 60 years in older patients and shows a bimodal distribution. ${ }^{8}$ It is more common in females and involves mostly the cervical spine. ${ }^{8}$

FCE is usually initially mistaken for transverse myelitis on MRI. It may be suspected in the absence of typical findings of inflammatory, infectious, or autoimmune etiologies on a detailed work-up. Currently, it can be diagnosed only by histopathology in postmortem cases or suspected on clinical grounds according to the criteria laid down by AbdelRazek et $\mathrm{al}^{2}$ in a review. The first step is to establish the presence of myelopathy, followed by exclusion of traumatic or compressive etiology clinically or by imaging. Our case had a clinical picture of myelopathy without any history of significant trauma or signs of compressive etiology on clinical examination and MRI. The next step is to rule out any inflammatory etiology. In our case, there was no pleocytosis or raised IgG index on CSF study, and there was also an absence of gadolinium enhancement on MRI, suggestive of a noninflammatory condition. CSF proteins may be slightly raised in cases of infarction. The next step is to establish the diagnosis of spinal infarction. Accordingly, there was a vascular distribution of spinal cord involvement on clinical examination with sparing of posterior column sensations and on MRI with predominant involvement of the ASA territory as opposed to inflammatory conditions. Other minor criteria supporting the diagnosis were

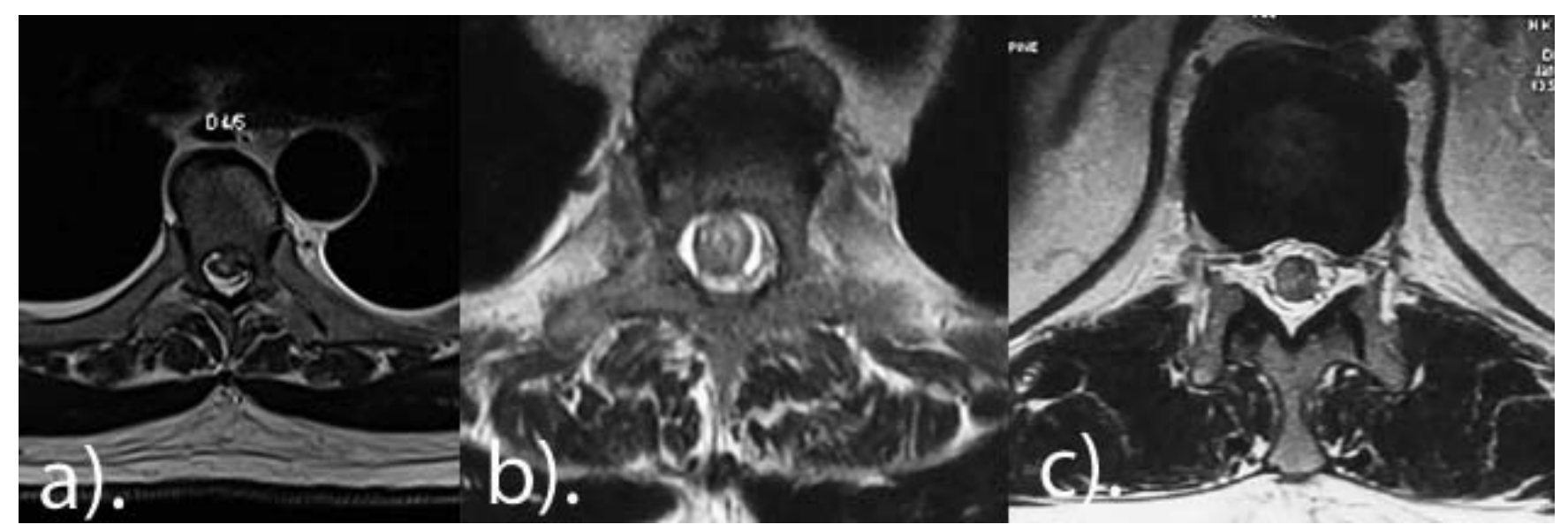

Figure 2. Axial T2 magnetic resonance image at T4-T5 showing left-sided paracentral disc protrusion indenting the thecal sac (a). There is predominant involvement of the anterior part of the cord in anterior spinal artery distribution, also seen at T5-T6 (b) and T10-T11 (c). 


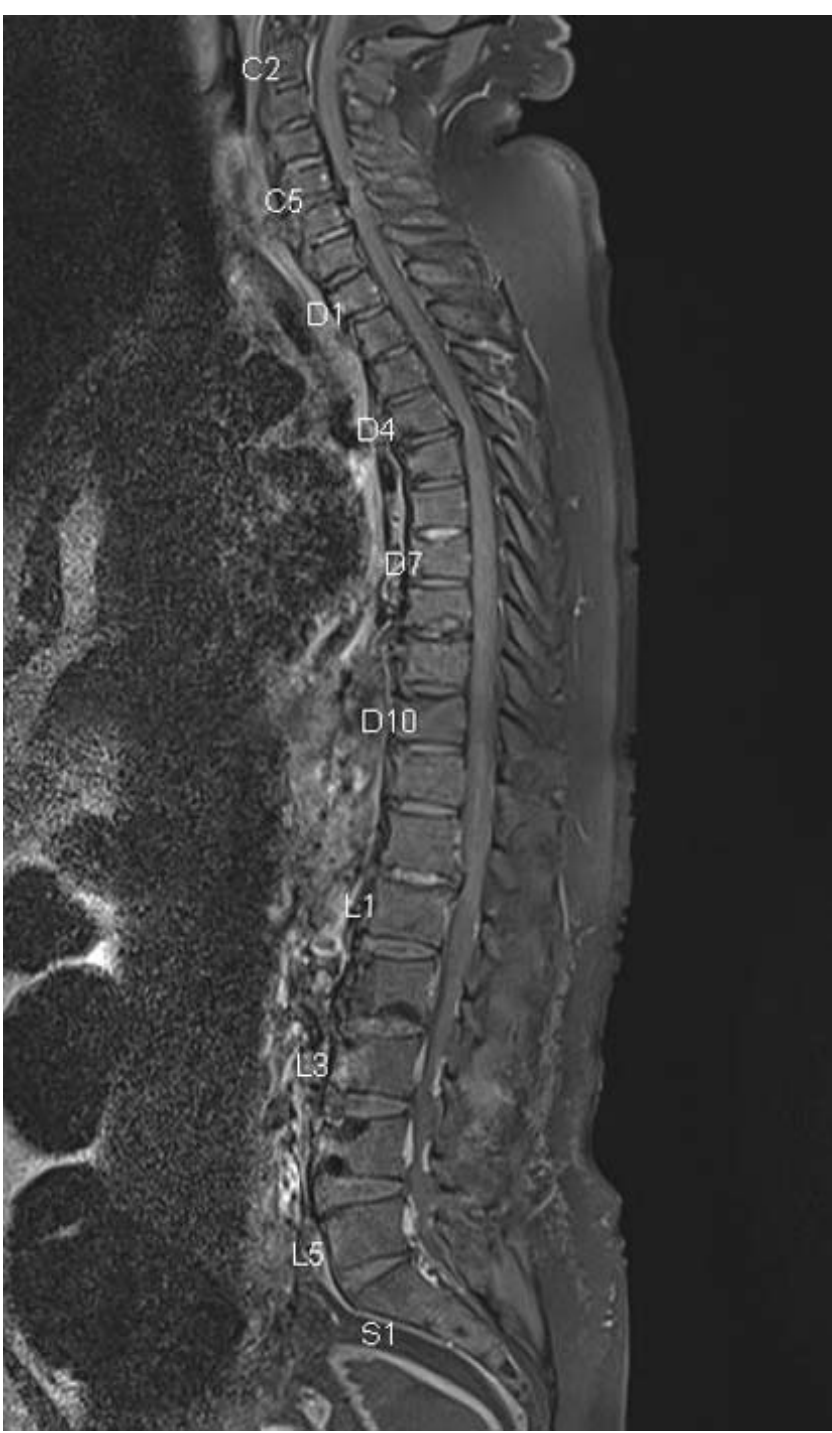

Figure 3. Postgadolinium sagittal MRI of the spine showing no abnormal cord enhancement and no evidence of leptomeningeal enhancement, ruling out inflammatory etiology.

the presence of new onset of back pain after a history of a trivial fall and symptom progression reaching a nadir within 8 hours. In inflammatory conditions, there may be a prodromal illness, and symptom onset may be slow to develop. There was temporal relationship to trivial trauma and presence of degenerated disc at T4-T5 corresponding to the highest level of spinal cord infarction on MRI. Finally, a high likelihood of FCE is established in the absence of major vascular risk factors, such as aortic pathology, diabetes mellitus, smoking, hypertension, prior vascular episodes, peripheral vascular disease, or age of more than 60 years. Although not included in the criteria, the presence of cord enhancement on DWI and corresponding hypointensity on ADC further consolidates the diagnosis of spinal cord infarction. ${ }^{9}$ Hence, these investigations should be included in the criteria. ${ }^{9}$ Digital subtraction angiography is not required to establish the diagnosis of FCEM according to the criteria by AbdelRazak et $\mathrm{al}^{2}$ and hence was not done. Additionally, we found no abnormality on blood picture for autoimmune etiologies, such as multiple sclerosis, NMO, systemic lupus erythematosus, Sjogren syndrome, or antiphospholipid antibody syndrome.

The artery of Adamkiewicz is the largest medullary feeder to the ASA in the mid- and lower sections of the spinal cord. ${ }^{10}$ This vessel is usually located at the T9-T12 levels, but in $15 \%$ of the population, it may arise from $\mathrm{T} 5$ to $\mathrm{T} 8{ }^{10}$ It is usually a single artery with a left-side origin. ${ }^{10}$ Hence, in our case, there may be involvement of this artery, as the thoracic disc T4-T5 was mainly left paracentral. The mechanism of FCE is not fully understood but has been proposed by AbdelRazek et al. $^{2}$ A portion of nucleus polposus may get separated after a sudden increase in intradiscal pressure, such as a trivial fall, and may gain entry into the spinal vasculature through the neovascularization channels in the degenerated disc. Other proposed pathways are vascular sinusoids adjacent to Schmorl's nodes and abnormal persistence of vascular channels in the disc in adults. The embolic material may travel retrograde to the radicular artery and then lodge along the blood flow into the spinal artery, causing infarction. A venous route involving normal anterograde travel to the vena caval system followed by retrograde travel into the Batson venous plexus has also been proposed. This migration is aided by an increase in the intrathoracic or intra-abdominal pressure during daily activities.

Thoracic disc herniation causing a direct extrinsic compression of the arterial supply of the cord is another possibility that should be taken into consideration. Few literature case reports in English are available describing such presentation. ${ }^{11,12}$ However, in these reports, the neurological deficit was asymmetrical and transient, as it gradually improved over time with steroids. In our case and also in previously described case reports due to FCEM, the neurological deficit did not improve over time even with steroids and often led to death or permanent paralysis. Extrinsic compressive etiology in our case is unlikely due to the absence of any associated radicular pain, symmetrical neuro- 


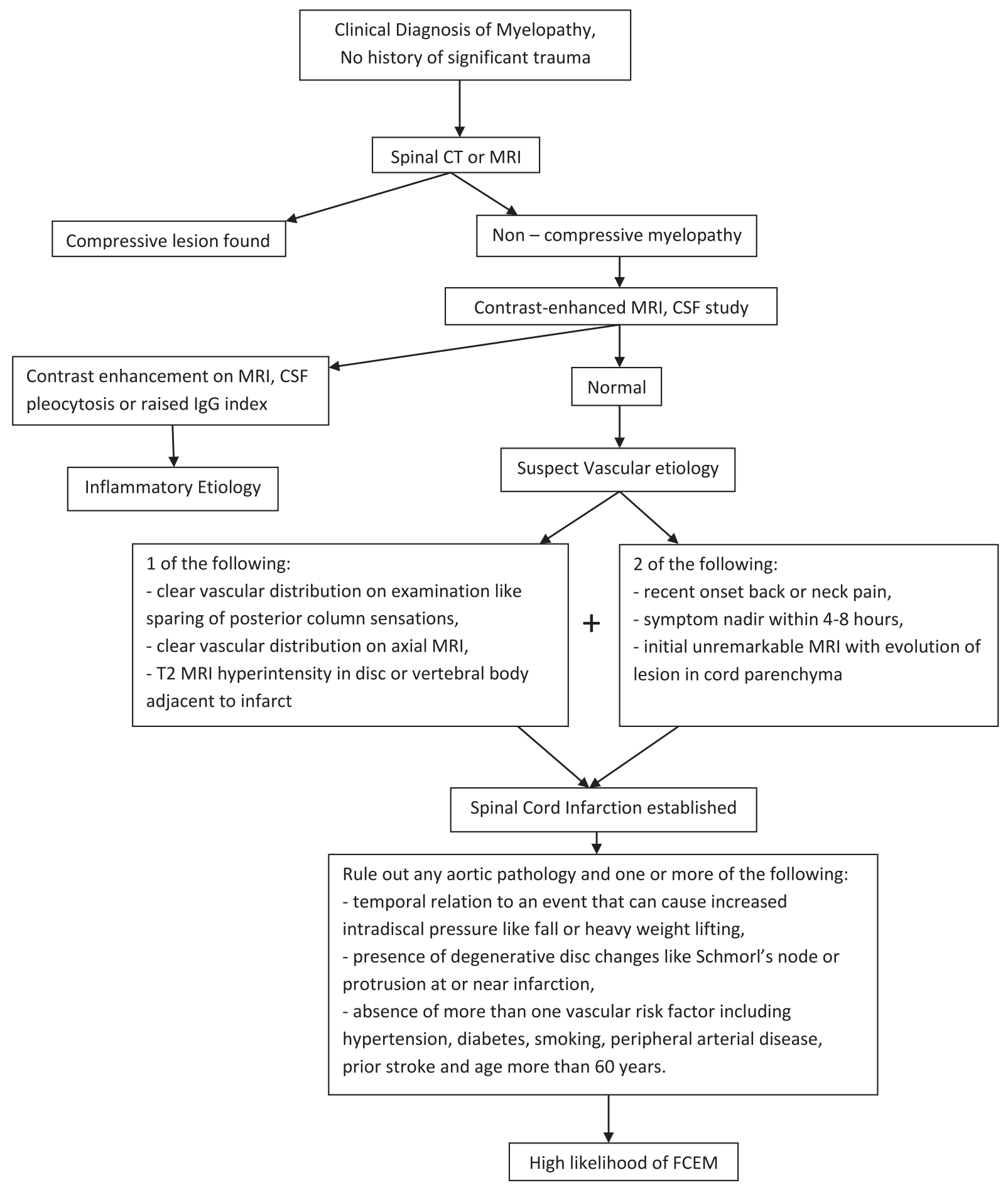

Figure 4. An algorithmic approach to diagnosing fibrocartilaginous embolic myelopathy.

logical compromise on both sides of the body, and failure to show improvement with steroids.

Currently, there are no definite treatment options available for FCEM., ${ }^{2,7}$ Methylprednisolone, intravenous immunoglobulins, and plasmapheresis have been tried, suspecting transverse myelitis, but with no improvement. ${ }^{2,7}$ Thrombolytic therapy or surgi- cal treatment has not been mentioned in the existing literature to have any role. ${ }^{2,7}$ The only viable option is rehabilitation. ${ }^{2}$ The overall prognosis for neurological recovery is dismal. ${ }^{2}$

Hence, we report a rare case of myelopathy possibly due to embolism of fibrocartilaginous material from the nucleus polposus causing ischemia 
in ASA territory. Although the condition is underdiagnosed, it should be suspected in cases with MRI images of transverse myelitis and in whom no other cause can be identified. Criteria are available to diagnose FCEM on clinical grounds. Currently, there are no medical or surgical treatment options available for this condition, and the prognosis for neurological recovery remains poor.

\section{ACKNOWLEDGMENTS}

The authors would thank the patient and his family to give consent for reporting of this case.

\section{REFERENCES}

1. Naiman JL, Donohue WL, Prichard JS. Fatal nucleus pulposus embolism of spinal cord after trauma. Neurology. 1961;11(1):83-87.

2. AbdelRazek MA, Mowla A, Farooq S, Silvestri N, Sawyer R, Wolfe G. Fibrocartilaginous embolism: a comprehensive review of an under-studied cause of spinal cord infarction and proposed diagnostic criteria. J Spinal Cord Med. 2016;39(2):146-154.

3. De Risio L. A review of fibrocartilaginous embolic myelopathy and different types of peracute non-compressive intervertebral disk extrusions in dogs and cats. Front Vet Sci. 2015;2(8):24.

4. Cauzinille L, Kornegay JN. Fibrocartilaginous embolism of the spinal cord in dogs: review of 36 histologically confirmed cases and retrospective study of 26 suspected cases. J Vet Intern Med. 1996;10(4):241-245.

5. Rubin MN, Rabinstein AA. Vascular diseases of the spinal cord. Neurol Clin. 2013;31(1):153-181.

6. Cheshire WP, Santos CC, Massey EW, Howard JF Jr. Spinal cord infarction: etiology and outcome. Neurology. 1996;47(2):321-330.

7. Moore BJ, Batterson AM, Luetmer MT, Reeves RK. Fibrocartilaginous embolic myelopathy: demographics, clinical presentation, and functional outcomes. Spinal Cord. 2018;56(12):1144-1150.

8. Shah S, Bryant P. Fibrocartilaginous emboli in the pediatric population: the role of rehabilitation in facilitating functional recovery. J Pediatr Rehabil Med. 2018;11(1):53-56.

9. Manara R, Calderone M, Severino MS, et al. Spinal cord infarction due to fibrocartilaginous embolization: the role of diffusion weighted imaging and short-tau inversion recovery sequences. J Child Neurol. 2010;25(8):1024-1028.

10. Lindeire S, Hauser JM. Anatomy, back, artery of Adamkiewicz. https://www.ncbi.nlm.nih.gov/books/NBK532971. Accessed Dec 6, 2018.

11. Guest JD, Griesdale DE, Marotta T. Thoracic disc herniation presenting with transient anterior spinal artery syndrome. A case report. Interv Neuroradiol. 2000;6(4):327-331.

12. Reynolds JM, Belvadi YS, Kane AG, Poulopoulos M. Thoracic disc herniation leads to anterior spinal artery syndrome demonstrated by diffusion-weighted magnetic resonance imaging (DWI): a case report and literature review. Spine J. 2014;14(6):e17-e22.

Disclosures and COI: The corresponding author affirms that he participated in and contributed sufficiently to the work and takes public responsibility for conception and design, data acquisition, analysis of data, and drafting of the manuscript. The other author played a significant role in critical revision, case management, administrative support, and supervision. No financial assistance was received in support of this study. The authors declare that they have no conflict of interest.

Corresponding Author: Mayank Gupta, MS, Department of Spine Surgery, Indian Spinal Injuries Centre, Sector C, Vasant Kunj, Delhi, India, PIN 110070. Phone: +91 9958338852; Email: id: mynk_vmmc@yahoo.in.

Published 30 June 2020

This manuscript is generously published free of charge by ISASS, the International Society for the Advancement of Spine Surgery. Copyright (c) 2020 ISASS. To see more or order reprints or permissions, see http://ijssurgery.com. 